\title{
Influence of the ground surface texture on the body balance of young people during natural position
}

\author{
Paulina Zofia Balińska ${ }^{1}$, Michał Kuczyński ${ }^{1}$ \\ ${ }^{1}$ Faculty of Physiotherapy, University School of Physical Education in Wroclaw, Poland \\ Correspondence to: Paulina Zofia Balińska, email: paulina.balinska@onet.pl \\ DOI: https://doi.org/10.5114/phr.2021.111811
}

Received: 18.02.2021 Reviewed: 06.04.2021 Accepted: 06.04.2021

\begin{abstract}
Background: One of the factors ensuring proper human functioning is the efficiency and control of the postural balance. The proper functioning of the vagus, the visual and vestibular systems, and proprioceptive receptors is achieved by appropriate and precise coordination of their work by the cerebellum. Constant contact with a surface while standing leads to information for the body orientation in space. Textured ground surfaces can be used as a very good tool for functional training and stimulation of the balance system.
\end{abstract}

Aims: This study aimed to evaluate the effect of different ground surface textures on the body balance of young subjects during natural position with eyes closed.

Material and methods: A total of 12 college students between the ages of 20 and 25 participated in the study. The subject was standing on a posturographic platform, compatible with a computer and suitable software. Four closed-eye trials were conducted on four different grounds: hard, fine mesh, coarse mesh, and tabbed pad. The duration of each measurement was 20 seconds.

Results: There was a ground-plane interaction for three COP parameters: fractal dimension, entropy, and frequency. Their values increased only in the frontal plane on mesh ground, with the greater increase for coarse mesh.

Conclusion: The nature of the contact surface between the foot and the shoe insert influences the stability of the standing posture and depends on the shape, number and size of the contact points.

Key words

postural stability, posturography, postural control, mechanoreceptors. 


\section{Introduction}

The ability to maintain body balance is crucial for its psychomotor development, basic activities in daily life, physical activities, and the rehabilitation process. Therefore, one of the very important factors ensuring the proper functioning of humans is the efficiency and effectiveness of the balance control system. Postural balance is when the body maintains an upright posture and proper body orientation in space by balancing the forces acting on the body $[1,2]$.

Maintaining body balance depends on the proper functioning of the postural control system, whose role is to maintain the centre of gravity (COG) in the sagittal and frontal planes within a field of support defined by the contours of the feet. A significant role in postural control is played by the coordination and processing of incoming information from the visual and vestibular system and the proprioceptors of muscles, tendons, joint capsules, and skin. The proper functioning of the vagus, the visual and vestibular systems, and the proprioceptors is ensured by the precise coordination of the cerebellum. The sense of balance is located in the membranous labyrinth of the inner ear. The ability to maintain body equilibrium occurs automatically and is controlled by the spinal cord, brainstem, and cortical areas of the brain $[3,4]$.

Factors that may cause balance disorders include the influence of some motor activity, misalignment of the foot, unexpected auditory, motor, or sensory stimuli, or the type of ground surface. However, balance problems may not always indicate a pathology [4]. For example, in a relaxed standing position, the body deviates from the vertical, which is taken as an indication of constant activity and dynamics of the balance system [5]. These unnoticeable movements are primarily based on the sensorimotor information received from the plantar surface of the foot [6]. Constant contact during standing position with the surface leads to the acquisition of information aimed at the orientation in space [7,8]. In response to the acquired somatosensory information, plantar muscles are activated, initiating a corrected postural response [9].

The most objective methods for assessing the functioning of the balance system are posturographic tests using a platform with a computer system. Then, the analysis of postural control takes place in a standing position and is subjected to analysis and measures of the COG displacements [5]. In many experiments, researchers have observed that textured surfaces can be used as a very good tool for functional and gait training for improving the functioning of the balance system.

\section{Aims}

This study aimed to evaluate the effect of different ground surface textures on the body balance of young subjects during a natural position with the eyes closed. Based on the literature, two assumptions were made: (1) COP measurement is an adequate method to assess body balance, and (2) the possible effect of ground texture on body balance depends on the geometry of direct foot contact with the ground.

\section{Material and methods}

The study was conducted in November 2019 at the University School of Physical Education in Wroclaw, Poland. A total of 12 students between the ages of 20 and 25 participated in the study. There were five females and seven males in this group. The students who participated in the study were given detailed information about the study and gave written consent. Subjects who did not have any neurological conditions or diseases related to the musculoskeletal and cardiovascular systems were eligible for the study. Athletes were excluded from the study. The detailed characteristics of study subjects are presented in tables 1 and 2. 
Table 1. The number of the students studied.

\begin{tabular}{|c|c|c|}
\hline Sex & Women & Men \\
\hline Age [years] & $N=5$ & $N=7$ \\
\hline 20 & 0 & 2 \\
\hline 21 & 0 & 1 \\
\hline 22 & 0 & 1 \\
\hline 23 & 1 & 1 \\
\hline 24 & 4 & 1 \\
\hline 25 & 0 & 1 \\
\hline
\end{tabular}

Table 2. Bodyweight and height of the students studied.

\begin{tabular}{|l|c|c|c|c|c|}
\hline & Mean & SD & Min & Median & Max \\
\hline Total & 69.5 & 14.3 & 46 & 74.5 & 89 \\
\hline Total & 175.6 & 11.5 & 154 & 178 & 190 \\
\hline
\end{tabular}

The research subject assumed a regular, relaxed, and habitual standing position with upper limbs and the torso on a posturographic platform compatible with a computer and suitable software (Fig. 1). Subjects were standing barefoot on the device, and both feet were positioned according to a designated template placed on the platform. Four trials were performed with eyes clo-

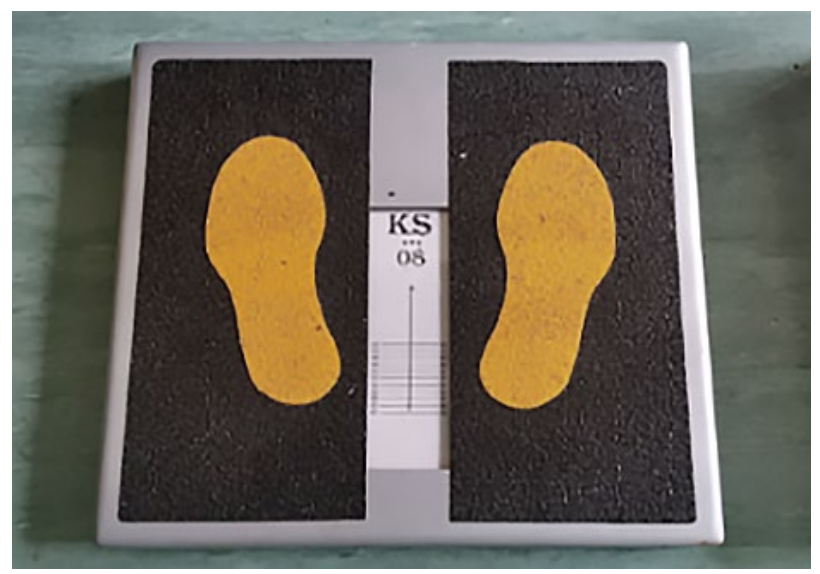

Figure 1. Posturographic platform. sed, during which the COG displacements were recorded using the software. The first measurement was performed standing with both feet on the platform in a natural position (Fig. 2), the second in a standing position on a fine mesh (Fig. 3), the next on a coarse mesh (Fig. 4), and the last on a tabbed pad (Fig. 5).

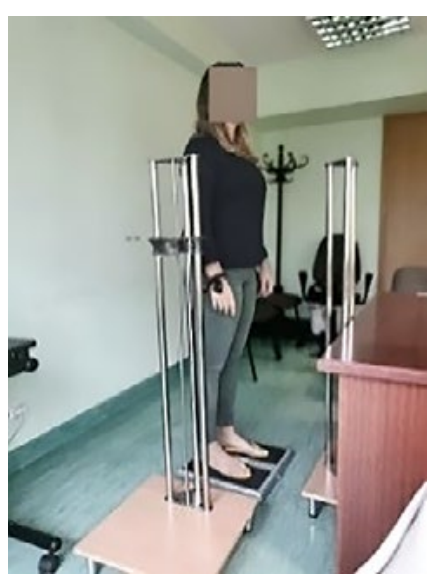

Figure 2. Double-leg standing position on the platform with eyes closed. 

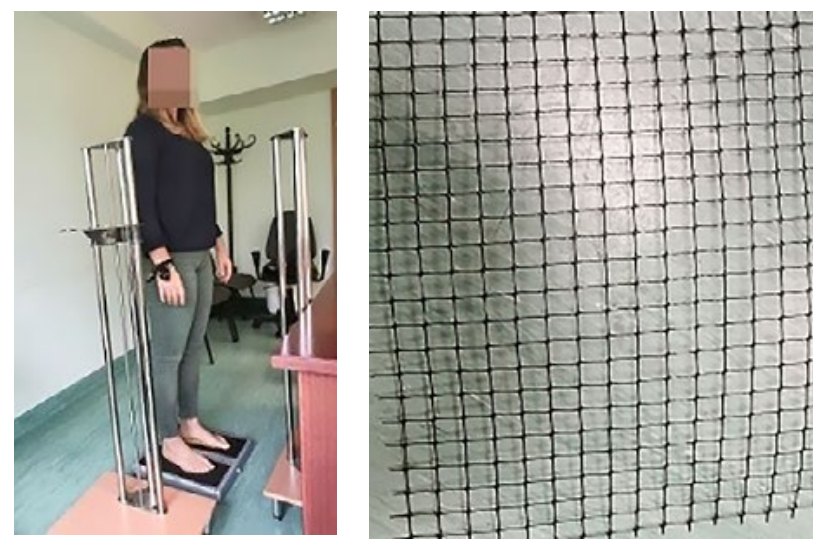

Figure 3. Double-leg standing position on a platform with fine mesh.
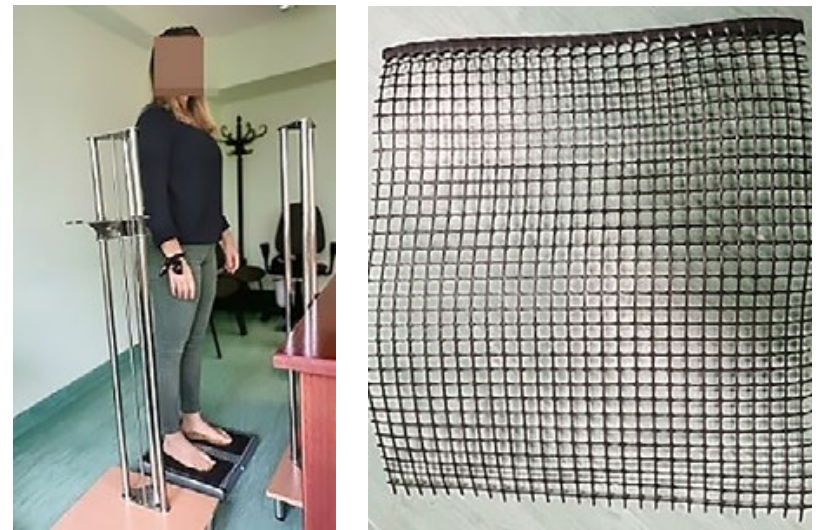

Figure 4. Double-leg standing position on a platform with coarse mesh.
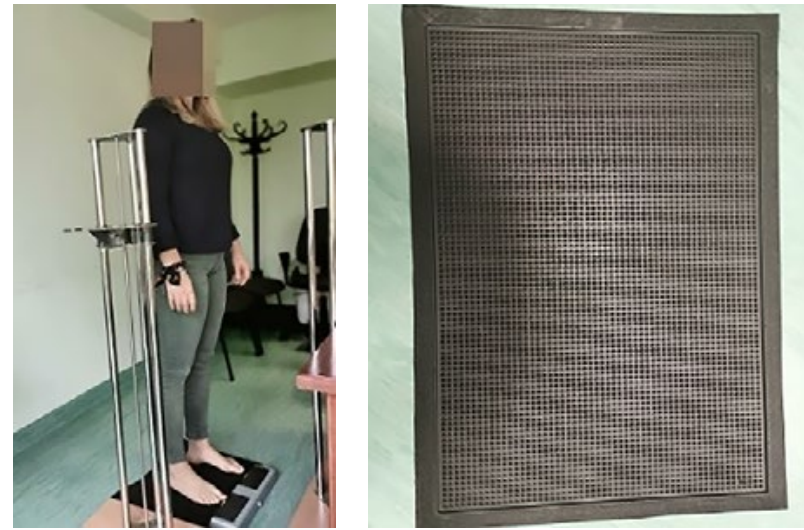

Figure 5. Double-leg standing position on a platform with tabs.
Before the test, the subject made postural corrections to keep the cursor within a centrally placed stationary square. The duration of each measurement was 20 seconds. The order of testing was randomized. All trials were conducted between 9:00 a.m. and 1:00 p.m., with adequate daylight and calm conditions. Body height and weight were also measured, and BMI was calculated from these measurements.

All tested dependent variables describing the properties of the balance system showed normal distribution. Therefore, analysis of variance with the repeated ANOVA $(4 \times 2)$ was applied for four different types of ground surfaces and both planes simultaneously. When significant differences were found, Fisher's NIR post-hoc tests were performed comparing the results on the experimental grounds with the results obtained under natural conditions. For individual comparisons, Cohen's effect size was calculated using standard values of 0.2 as the limit for a small effect, 0.5 for a medium effect and 0.8 for a large effect. Results are given graphically as mean $\pm 95 \%$ confidence intervals. STATISTICA software was used to analyze the results. The significance level was considered for $\alpha<0.05$.

To avoid uncertainty, the time series or centre of pressure (COP) signal is defined as the centre of pushing or, more specifically, as the point of application of the resultant forces acting on the ground, which are the result of the correction and stabilization reactions of a person standing on this ground (force platform). It is also worth explaining two calculated parameters of these series: fractal dimension and entropy of the COP.

The fractal dimension of the COP describes the complexity of this signal. A higher value corresponds to greater complexity, which is equated in the literature with better adaptability to the environment and unexpected challenges to the balance system. This is usually combined with better use of sensory information and increased degrees of freedom involved in maintaining balance. This applies to both biomechanical and 
sensorimotor degrees of freedom. On the other hand, smaller fractal dimension values indicate greater stability, but at the expense of the benefits mentioned above. Thus, a certain compromise between adaptability and stability occurs here. Therefore, only a thorough analysis of the subject's abilities combined with the degree of task difficulty allows for a reasonable interpretation.

The entropy of the COP describes the irregularity of this signal, with high entropy being a more irregular signal. More irregular COPs occur with less conscious attention and correspond to increased automaticity in balance regulation. Typically, high entropy of COP is observed in well-controlled postural tasks and physically active individuals. One special case is dual tasks, i.e., combining a balance task with an additional task (either cognitive or motor). This additional task requires attention that is taken away from the postural task. As a result, the entropy of COP usually increases, accompanied, especially in young people, with a decrease in amplitude. Reduced entropy of COP is often recorded in individuals with balance deficits. This indicates a need for greater attentional involvement to ensure adequate stability, i.e., a decrease in the automaticity of balance regulation in these individuals.

\section{Results}

For the amplitude of COP, the main plane effect was observed: $F(1,11)=73.46(p<0.0001)$, indicating significantly greater body sway amplitude in the sagittal plane. There was no main effect of the ground or ground-plane interaction (Fig. 6).

For the velocity of COP, the main plane effect was noted: $F(1,11)=32.877 \quad(p=0.00013)$. Mean velocity COP was significantly higher in the sagittal plane than in the frontal plane. There was no main effect of ground or ground-plane interaction (Fig. 7).

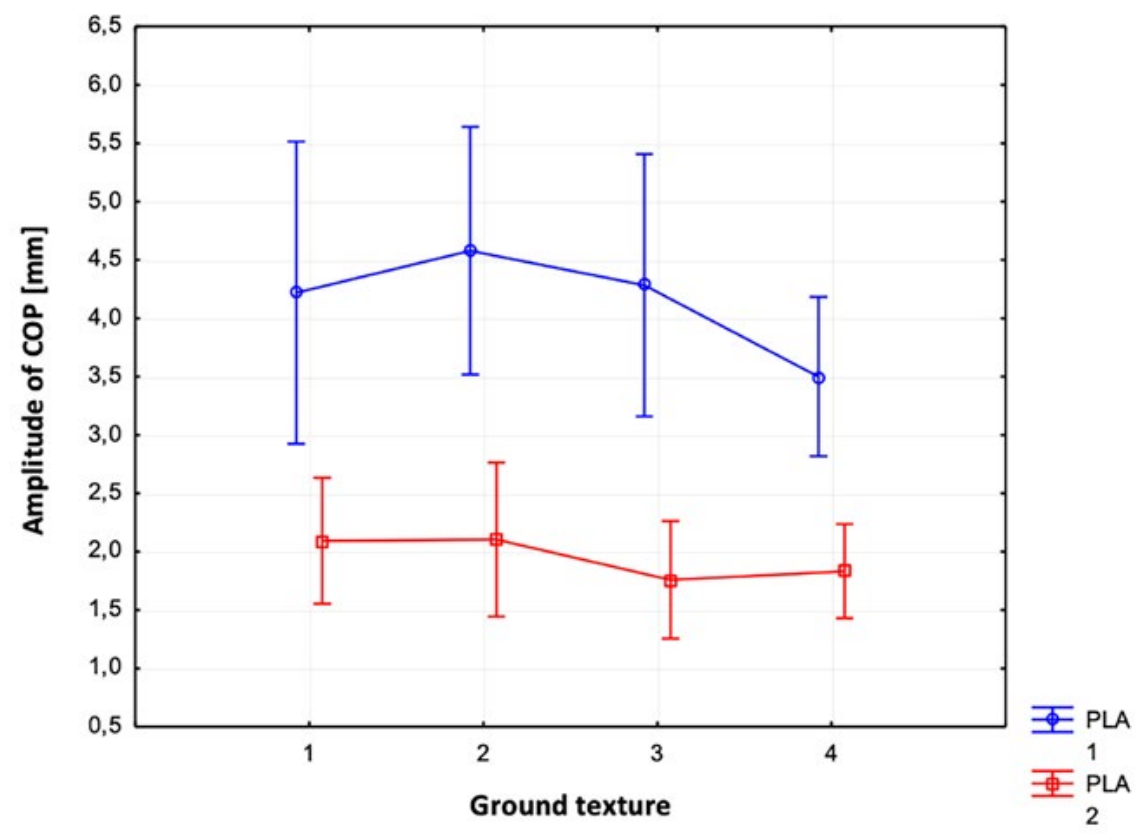

Figure 6. Relationship between the ground surface and amplitude of COP in the sagittal (1) and frontal (2) planes considering ground texture: neutral (1), fine mesh (2), coarse mesh (3), and tabbed pad (4). 


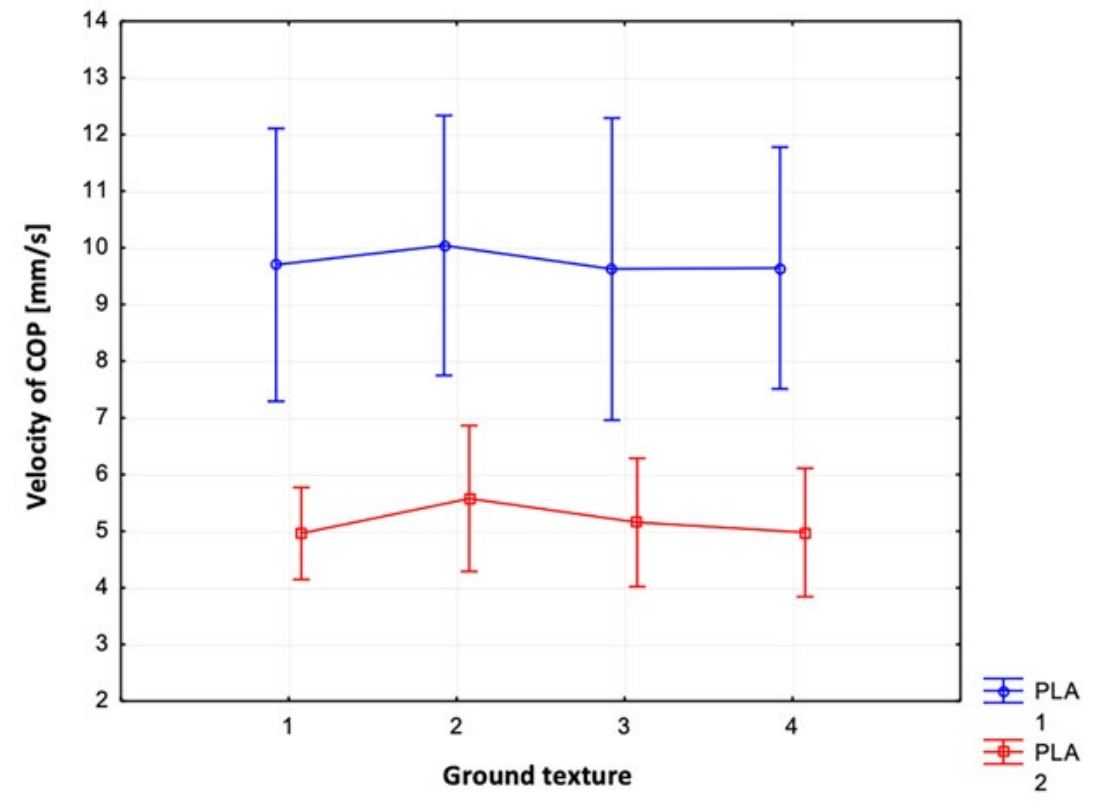

Figure 7. Relationship between the ground surface and velocity of COP in the sagittal (1) and frontal (2) planes considering ground texture: neutral (1), fine mesh (2), coarse mesh (3), and tabbed pad (4).

However, there was a ground-plane interaction for the fractal dimension: $F(3,33)=4.197,(p=0.013)$. A post-hoc test indicated an increase in fractal dimension in the frontal plane between the first neutral ground and the third on the coarse mesh $(p<0.0007)$ and the second on the fine mesh $(p<0.03)$ (Fig. 8).

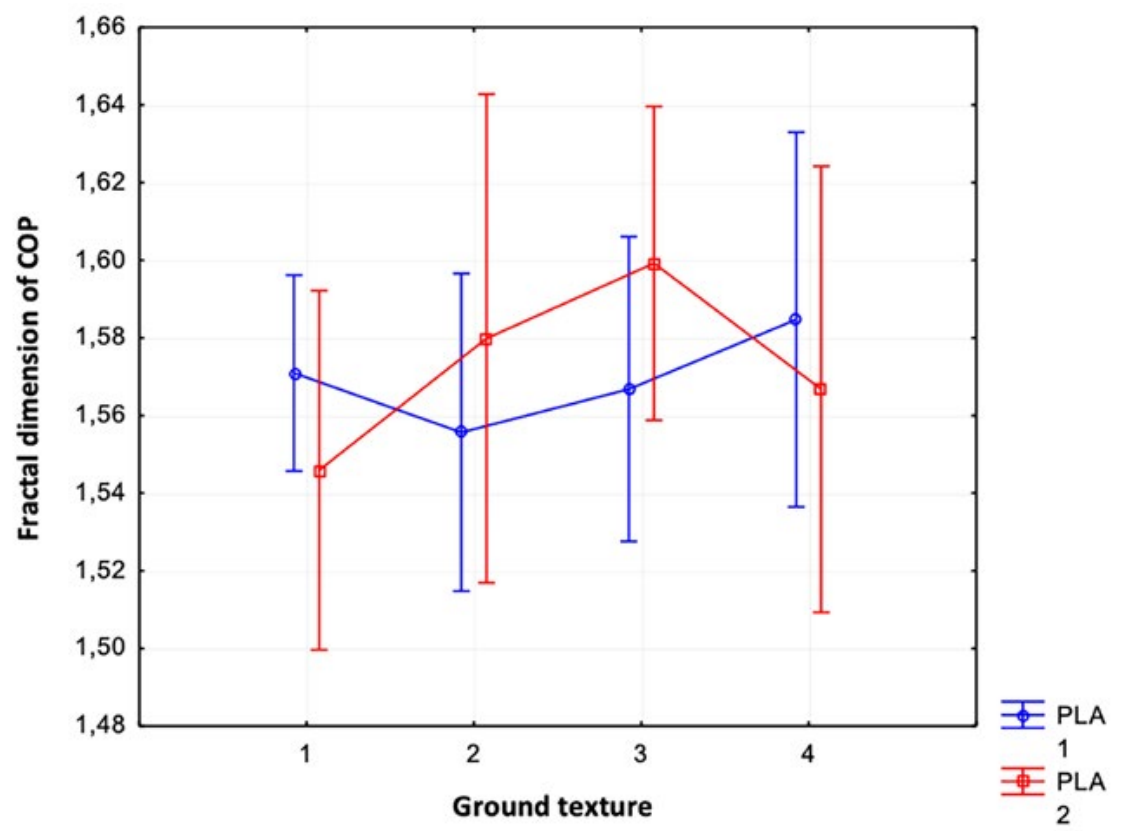

Figure 8. Relationship between the ground surface and fractal dimension of COP in the sagittal (1) and frontal (2) planes considering ground texture: neutral (1), fine mesh (2), coarse mesh (3), and tabbed pad (4). 
For the entropy of COP, there was a ground-plane interaction: $F(3,33)=5.672(p=0.003)$. A post-hoc test indicated an increase in entropy between standing on hard ground and standing on a coarse mesh $(\mathrm{p}<0.0001)$ (Fig. 9).

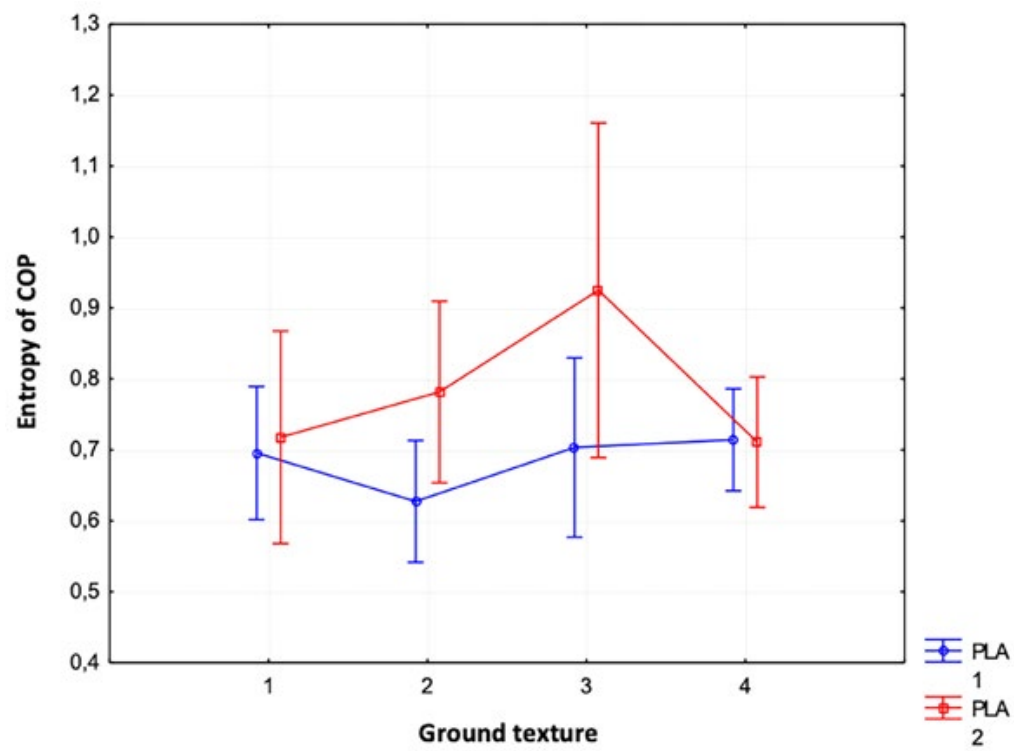

Figure 9. Relationship between the ground surface and entropy of COP in the sagittal (1) and frontal (2) planes considering ground texture: neutral (1), fine mesh (2), coarse mesh (3), and tabbed pad (4).

For the mean frequency of COP, a ground-plane interaction also emerged: $F(3,33)=3.596,(p=0.0236)$. The post-hoc test indicated, as before, changes in the frequency of COP only in the frontal plane. There was an increase in the frequency of COP on coarse mesh $(\mathrm{p}=0.0043)$ and fine mesh $(\mathrm{p}=0.0360)$ ground surfaces (Fig. 10).

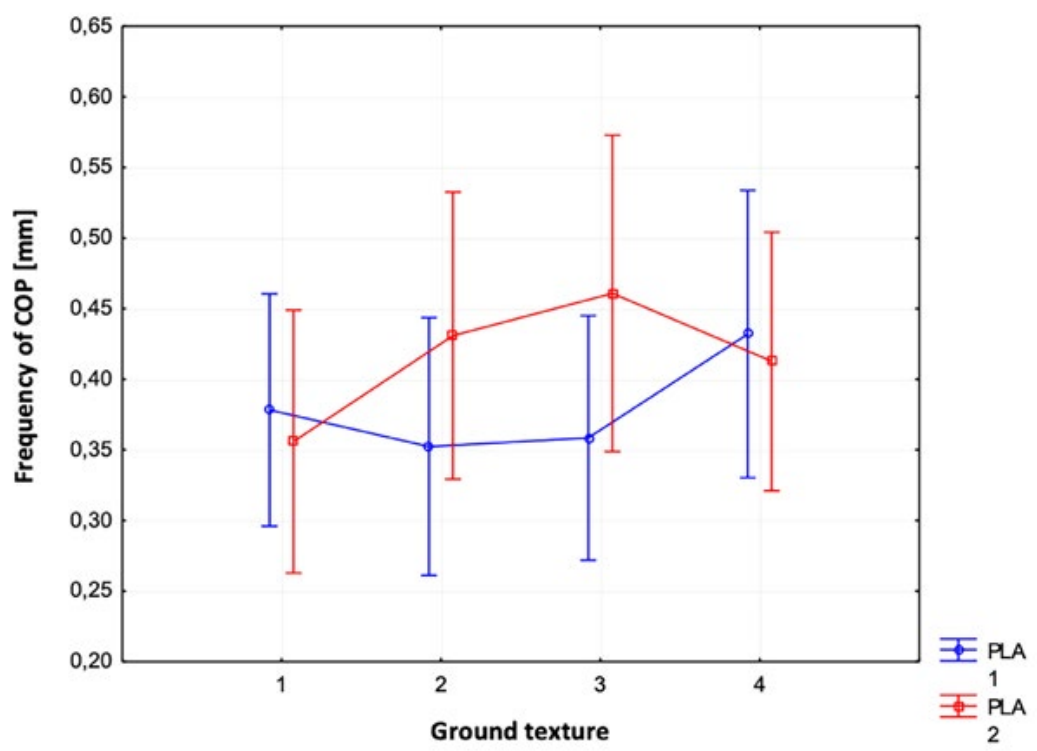

Figure 10. The relationship between the ground and the frequency of COP in the sagittal (1) and frontal (2) planes considering ground texture: neutral (1), fine mesh (2), coarse mesh (3), and tabbed pad (4). 
Table 3 shows Cohen's effect size values for comparisons of $\mathrm{COP}$ parameters that showed significant differences between the hard ground and the experimental substrates used in this study. The effect si- zes allow for a better assessment of the significance of the observed differences from a practical or clinical perspective.

Table 3. Cohen's effect size for selected comparisons of COP parameters in the frontal plane.

\begin{tabular}{|l|c|c|}
\hline & Fine mesh / Hard ground & Coarse mesh / Hard ground \\
\hline Fractal dimension & 0.38 & 0.79 \\
\hline Entropy & 0.28 & 0.65 \\
\hline Frequency & 0.53 & 0.70 \\
\hline
\end{tabular}

\section{Discussion}

This study aimed to evaluate the influence of different ground surface textures on the body balance of young people standing in a natural position with eyes closed. The most important of the results obtained is a ground-plane interaction for the three COP parameters, proving their increase only in the frontal plane. No changes were noted in the sagittal plane. In particular, there was an increase in the fractal dimension, which may indicate better adaptability of the balance system to the surrounding external environment. An increase in entropy was also observed, and as a result, an increase in automaticity and efficiency of balance regulation, which is characteristic of postural control with less involvement of attention and participation of consciousness. Therefore, the balance system optimizes its performance. An increase in mean frequency was also observed, which may have implications for the mechanisms responsible for automatic postural control. All of the observed changes occurred on the mesh-textured grounds, with the effect size for the coarse mesh being medium (close to large), while the effect size for the fine mesh was lower (between small and medium). The tabbed ground did not affect the balance to any significant degree.

The results obtained in our study are consistent with the analysis of the study by Qiu et al. [10], during which it was observed that standing on the textured surface of a shoe insert could reduce medial-lateral postural sway in healthy older adults. It should be noted that textured inserts increased balance control only in older adults; nevertheless, the number of subjects studied was small. Noteworthy are the results obtained from another study by Qiu et al. [12], during which it was shown that standing position with eyes closed on a textured surface ground has a positive effect on the anterior-posterior COP displacements. These findings may suggest that textured insoles may lead to the activation of mechanoreceptors on the plantar surface of the foot and feedback to the central nervous system and represent an improvement in the postural control system [11,12].

The opposite results were obtained by Hatton et al. [13], who showed no significant effect of textured inserts during measurements with the eyes open and closed on the studied parameters in both the sagittal and frontal planes. The discrepancy in these results may be due to differences regarding the subjects' numbers and age differences. The balance regulation system may show significant deficits. It should also be noted that the different results may be due to the various implementation of the experimental interventions under different conditions, with the use of textured ground surfaces, but also the use of shoe inserts. 
Some previous studies have shown that textured ground surfaces or insoles do not improve body balance. This may be due to the characteristics of the type of ground or orthotics used, more specifically the material from which they are made, the geometric pattern and other characteristics such as height and width [14]. In addition, many other factors can also affect body balance, such as genetic conditions, psychological problems, external environment, postural defects, and low physical activity and sedentary lifestyle.

An analysis of our findings and those of other researchers indicates that most of the findings relate specifically to experiments in which inserts or textured surfaces were applied for the first time. It is unknown whether a familiarization period is required, how long it can take, and whether habituation to texture stimuli can significantly affect the postural control system.

Nevertheless, most studies provide evidence that improved information stimulating mechanoreceptors can positively affect the balance system function. It is believed that human postural control is modulated based on the integration of somatosensory data, including vision, deep sensation, and vestibular system coupling $[15,16]$. Therefore, the presented results can provide a strategy for physiotherapeutic management and support future research regarding the effectiveness of various types of ground on maintaining balance in patients with postural control disorders.

A limitation of the present study may be the relatively small number of subjects. Thus, it is necessary to continue the study in a larger experimental group towards detecting possible and potential effects after regular and prolonged contact with a variety of ground textures. In addition, it is important to note that this study was conducted in a group of young and healthy individuals, so these results cannot be fully used to inform the improvement of balance ability in an older population with a higher risk of falls [16].
In summary, textured surfaces may be a feasible alternative, being an easily accessible and inexpensive way to improve balance performance. Further studies are needed to identify the most suitable inserts. The comfort and efficacy of different surface textures in supporting sensorimotor function need to be studied with a large and homogeneous group over a considerably longer period, including several months [12].

\section{Conclusion}

There was an increase in fractal dimension, entropy, and frequency of COP displacement in the frontal plane while standing on a coarse and fine mesh with the eyes closed. Activation of mechanoreceptors located in the plantar surface of the foot plays a crucial role in postural control, and closing the eyes enforces the balance control system to process much more proprioceptive information. In the frontal plane, the most important influence on the balance of young people during a natural position with the eyes closed is exerted by the ground with its coarse mesh. 


\section{References}

1. Kaźmierczak U, Kropkowska P, Zniszczol P, Radzimińska A, Strojek K., Goch A, et al. Evaluation of static balance with the use of the posturographic platform in poorly visionaries and blind persons. J Educ Health Sport. 2016;6(8):102-12.

2. Winiarska A, Ziółkowska A, Świtaj K, Wojtczak P. Balance of individuals at different age involved in physical activity - review of publications. J Educ Health Sport 2017;(7):978-85.

3. Henry M, Baudry S. Age-related changes in leg proprioception: implications for postural control. J Neurophysiol 2019;122(2):525-38.

4. Kowalik T, Srokowska A, Lewandowski A, Piekorz $\mathrm{Z}$, Zukow W. The influence of the sense of sight for a sense of general balance. J Educ Health Sport. 2015;5(11):720-6.

5. Kuczyński M, Podbielska M, Bieć D, Paluszak A, Kręcisz K. The basics of postural control assessment: what, how and why do we need to measure? Acta Bio-Opt Inform Med. 2012;18(4):243-9.

6. Viseux F, Lemaire A, Barbier F, Charpentier P, Leteneur S, Villeneuve P. How can the stimulation of plantar cutaneous receptors improve postural control? Review and clinical commentary. Neurophysiol Clin. 2018;49(3):263-8.

7. Picker J, Ratuszek-Sadowska D, Pyskir M, Pawlak-Osińska K, Szymkuć-Bukowska I, Hagner W. Assessment and comparison of selected parameters of posture stability in patients with balance disorders and healthy people by stabilometric analysis. J Educ Health Sport. 2016;6(12):230-42.

8. Kenny R, Eaves D, Martin D, Hatton A, Dixon J. The effects of textured insoles on quiet standing balance in four stance types with and without vision. BMC Sports Sci Med Rehabil. 2019;11(5):1-8.

9. Viseux F, Lemaire A, Barbier F, Charpentier P, Leteneur S, Villeneuve P. How can the stimulation of plantar cutaneous receptors improve postural control? Review and clinical commentary. Neurophysiol Clin. 2018;49(3):263-8.
10. Qiu F, Cole MH, Davids KW, Hennig EM, Silburn PA, Netscher H, et. al. Enhanced somatosensory information decreases postural sway in older people. Gait Posture. 2012;35(4):630-5.

11. Alfuth M. Textured and stimulating insoles for balance and gait impairments in patients with multiple sclerosis and Parkinson's disease: A systematic review and meta-analysis. Gait Posture. 2017;51:132-41.

12. Qiu F, Cole MH, Davids KW, Henning EM, Silburn PA, Netscher H, et.al. Effects of Textured Insoles on Balance in People with Parkinson's Disease. Plus One. 2013;8(12):1-8.

13. Hatton A, Dixon J, Rome K, Newton J, Martin D. Altering gait by way of stimulation of the plantar surface of the foot: the immediate effect of wearing textured insoles in older fallers. J Foot Ankle Res. 2012;5(11):1-6.

14. Orth D, Davids K, Wheat J, Seifert L, Liukkonen J, Jaakkola T, et.al. The Role of Textured Material in Supporting Perceptual-Motor Functions. Plus One. 2013;8(4):e60349.

15. Lin C, Roche JL, Steed DP, Musolino MC, Marchetti GF, Furman GR, et.al. Test-retest reliability of postural stability on two different foam pads. J Nat Sci. 2015;1(2):e43.

16. Kenny R, Atkinson G, Eaves D, Martin D, Burn N, Dixon J. The effects of textured materials on static balance in healthy young and older adults: A systematic review with meta-analysis. Gait Posture. 2019;71:79-86. 